\title{
2.4GHz ISM Band Radio Frequency Signal Indoor Propagation
}

\author{
Michal Kochláň, IEEE Student Member \\ Department of Technical Cybernetics \\ Faculty of Management Science and Informatics \\ University of Žilina \\ Univerzitná 8215/1, 01026 Žilina, Slovakia \\ Email: michal.kochlan@fri.uniza.sk
}

\author{
Juraj Miček, Peter Ševčík \\ Department of Technical Cybernetics \\ Faculty of Management Science and Informatics \\ University of Žilina \\ Univerzitná 8215/1, 01026 Žilina, Slovakia \\ Email: \{juraj.micek, peter.sevcik\}@fri.uniza.sk
}

\begin{abstract}
Indoor environment is from the point of the wireless communication an extremely hostile environment. Despite this fact, wireless sensor network applications in the indoor environment are very common. Having signal propagation in a real environment, without considering interferences from other sources, we meet (not only indoors) four basic phenomenons path-loss, reflection, diffraction and scattering. Each of these effects impact on the spread of the signal and contributes to attenuation and distortion at the receiver side. Detailed description of the electromagnetic wave propagation can theoretically obtain the solution of Maxwell's equations. However, this is too demanding and for practical cases unusable. In practice, to describe the signal propagation, the approximate models are used, which are often based on experimental results. This contribution includes case study on indoor radio frequency signal propagation at $2.4 \mathrm{GHz}$ ISM Band with related math, supported by implementation of the propagation models and experimental results.
\end{abstract}

\section{INTRODUCTION}

I NDOOR wireless sensor network (WSN) applications, despite unfavorable conditions of indoor radio signal propagation, constitute the very attractive area. As an introduction, let us mention at least some of the frequently occurring applications that the authors consider representative.

\section{A. Intelligent Buildings}

In recent decades, an increased attention to matters related to sustainable environment has been dedicated. A large part of the environmental problems is related to the energy consumption of the society. It is worth to emphasize that the effective regulation of the overall energy consumption of the society is able to achieve significant advances in the state of the environment [1].

One of the dominant energy consumption components is the operation of residential and non-residential buildings. According to [2], in 2012, the energy consumption of buildings in the U.S. was $39 \%$, followed by $32 \%$ energy consumption share of an industry and transportation sector with portion of $29 \%$ of the total consumption of the energy. In Europe, the situation is quite similar. In 2010, the buildings operation took

This work was supported by Centre of excellence for systems and services of intelligent transport II. ITMS 26220120050 supported by the Research \& Development Operational Programme funded by the ERDF.
$41 \%$ of total energy consumption, $32 \%$ in transportation and an industry energy consumption shared $25 \%$ [3].

Remark 1.1: Note that compared to the U.S., the energy consumption intended for buildings operation in Europe is slightly higher. Also, an interesting fact is that the annual energy consumption of the residential buildings is about $200 \mathrm{kWh} / \mathrm{m}^{2}$, non-residential buildings are characterized by higher annual energy consumption of about $300 \mathrm{kWh} / \mathrm{m}^{2}$.

Since 1990 , the energy consumption increases annually by around $0.6 \%$ in the residential sector and by around $1.5 \%$ in the non-residential sector [2], [3]. The presented statistical data show that the highest energy burden in the developed countries lies in operation cost of buildings. Based on the predictions presented in [2], it is expected that within the next 20 years, the energy consumption share of the buildings will not be reduced, just to the contrary, we can assume a slight increase in the energy consumption (increase in about $1 \%$ ).

Based on the above, it is clear that the operation of buildings consumes huge amounts of energy. Therefore, it is extremely important to address the issues of intelligent buildings control so that the user comfort and the effective energy sources utilization can be ensured.

In order to be able to reduce the energy consumption, it is necessary to know why, where and when the energy consumption occurs. Finding the answers to these questions is possible thanks to the new technologies in the field of ICT (Information and Communication Technologies) such as [4], [5] or [6]. Using new ICT means in buildings we get intelligent monitoring and control systems of the buildings that enable increasing user comfort while cutting energy and environmental burden. Buildings equipped with modern control and monitoring systems are often labeled as "intelligent" or "smart".

An extensive control, communication and monitoring system installed in modern intelligent buildings today is often divided into the following six subsystems [1]:

- Lighting control subsystem (lights, blinds, etc.);

- Heating, Ventilation and Air Conditioning (HVAC) subsystem - creates a psychometric chart to help determining the optimal environmental parameters;

- Security and safety subsystem (entrance authorization, 
fire alarm, personnel tracking, etc.);

- Metering subsystem (electricity, gas, water and other parameters with connection to the energy control);

- Indoor climate monitoring subsystem (temperature, humidity, dust, concentration of $\mathrm{CO}_{2}, \mathrm{NO}_{x}$ ("Green Building Monitor ${ }^{\mathrm{TM}}$ " - Siemens system to inform staff, clients and visitors about the energy consumption, environmental load of the building and about the state of the building indoor climate);

- Guest control subsystem (navigation, information for visitors, access control, motion tracking, etc.).

It is obvious that the provision of these subsystems implies the utilization of the latest technologies in the field of ICT. It is necessary to sense a large number of parameters in the intelligent buildings and based on the values, the actuator elements drive the building so that the optimal operation of the building is assured [1]. Sensors gathering the information create an extensive network with defined rules of communication. Nowadays, more and more, we meet the wireless communication technologies in the area of building control, which, in comparison to a wired network, is characterized by lower installation cost, higher flexibility and scalability [7]. Thus, even in the intelligent building environment, there are gradually used technologies known under the names of WSN or WSAN (Wireless Sensor and Actuator Networks).

\section{B. WSN in the Industry (Industrial Automation)}

Industrial applications represent another promising area of an efficient use of the WSNs [8]. Industrial control systems that integrate WSAN offer several advantages over conventional distributed control system [9], [10]. These are in particular easiness of the sensors' and actuators' installation, self-organization of the network, a simple modification, easy expandability of the network, efficient distributed and parallel data processing and lower cost compared to the conventional solutions [11], [12], [13]. There are also some disadvantages of wireless solutions, which we can find in the occurrence of interferences, not only in the industrial environment, unpredictable delays in packets, limited capacity of transmission channels and so on [9], [11]. However, the mentioned drawbacks, are not substantially limiting for the development and the implementation of WSN applications in industry. According to [9], it is essential to address, in particular, the following issues:

- Limited sources of the energy, the limitation of communication attributes, computing and storage capacity of the network nodes (channel capacity, limited communication range, etc.);

- Network operation in high interference industrial environments and related dynamic change of network topology;

- High demands on Quality-of-Service (QoS), especially requirements on packet deliverability in a defined time;

- Effective utilization of data redundancy to increase system reliability and accuracy of the status information of the controlled/monitored system;
- Effective addressing of the related issues to the error rate $\left(\mathrm{BER} \in\left\langle 10^{-2} ; 10^{-6}\right\rangle\right)$ and variable transmission capacity of individual transmission channels (adaptive modulation schemes, channel coding, etc.);

- Security in industrial applications is a key issue. It is necessary to protect communication from intentional active and/or passive attacks;

- Large-scale deployment and ad-hoc network functionality. Many industrial applications consist of a large number of randomly distributed nodes, so it is advantageous if the network is able to build autonomously the communication links and to control the communication among the nodes;

- Integration with other networks (e.g. the Internet). This point is very important from the perspective of effective control of the enterprise approach to the technological level, which represent also a WSN.

In the field of the industrial automation and in the context of WSAN implementation, there are arising new problems (distributed processing of variables, redundancy utilization to increase system reliability, problems with delayed packets), whose solutions are interesting from the theoretical and application point of view [9]. Based on the current development, a sharp increase in wireless solutions even in industrial applications is expected [14] [15], [16], [17].

\section{Health Applications and Senior Assistance Services}

Electronic health-care is a broad and interesting application area of WSNs. Let us mention a few representative examples of such WSN utilization [11]:

- Monitoring of vital signs and other selected parameters of patients in hospitals;

- Telemonitoring of the patients without hospitalization;

- Tracking patients, visitors and hospital staff;

- Indoor climate monitoring within the hospital premises;

- Controlling access to medicines and identification of time and kind of medication use through patient node(s).

WSN networks are able to monitor the behavior of older people and those with disabilities and enable to keep track of their health status without significant restrictions on their lives and quickly identify the signs of disease [18], [19]. Because of the above examples, it is clear that in health-care we meet growing number of interesting WSN applications indoors and outdoors - modern applications of WSN that monitor vital signs of a human body, track patients, monitor hospital environment, serve as medical access control systems and many more [20], [21].

Almost all mentioned applications have one thing in common, WSNs operate in indoor environment. Indoor environment is from the perspective of the wireless communication subsystems, an extremely hostile environment [7]. Despite this fact, WSN applications in indoor environments are highly desired.

Further investigations dealing with the properties of the indoor signal propagation assume the wireless sensor node based on Texas Instruments' (TI) CC2511 transceiver that is highly suitable for the indoor environment implementation. 
It is possible to list a number of other interesting and prospective application areas of WSNs related to the currently popular term "Internet of Things" (IoT), but due to the limited extent, we do not.

\section{INDOOR SIGNAL PROPAGATION}

If we consider wireless transmission channel for evaluation, we come to the conclusion that its features do not fully meet the media properties for reliable high-speed communication. The transmission channel is sensitive to noise, interference generated by the obstacles, communication distance, etc. [22]. Moreover, those adverse effects vary in time and space at random, as a result of the change in position of the receiver and/or transmitter and dynamic environmental changes.

Having signal propagation in a real environment, without considering interferences from other sources, we meet four basic phenomenons [22]. These are:

- Path-loss;

- Reflection;

- Diffraction;

- Scattering.

Each of these effects impact on the signal propagation and contributes to the path-loss and the distortion of the received signal. Answering the question of which of the abovementioned effects has the most significant impact on the quality of reception is not possible in general. The answer depends on the wavelength, the environment type and many other specific conditions. It is obvious that the electromagnetic waves do propagate in the real environment attenuated, reflected, diffracted and scattered from and by the terrain, buildings and other objects. The detailed description of the propagation of electromagnetic waves can be theoretically obtained by solving Maxwell's equations with constraints [23]. The detailed description is only possible in case of having all physical characteristics of the objects affecting the propagation in mind and implemented into the equations. Unfortunately, this is computationally demanding and in practical cases not usable. Therefore, in practice, approximate models, which are often based on experimental results, are used to describe the radio frequency wave propagation.

For determining the signal path-loss in the line-of-sight (LOS), a sufficient solution is the radio signal propagation model at outdoor environment that assumes that other propagation effects can be neglected [23].

Remark 2.1: Other propagation effects can be neglected when an open area transmitter and/or receiver antennas are placed at a sufficient height above the ground.

In this case, it is possible to determine the signal power at the receiving antenna out of the Friis equation:

$$
P_{r}=A_{e} S_{r}=\frac{\lambda^{2}}{4 \pi} G_{r} \cdot \frac{G_{t} P_{t}}{4 \pi d^{2}}=\left(\frac{\lambda}{4 \pi d}\right)^{2} G_{r} G_{t} P_{t},
$$

where,

$d$ is distance between the transmitter and the receiver;

$\lambda$ represents signal wavelength;

$P_{t}$ represents transmission power;
$P_{r}$ represents power at the receiving antenna;

$G_{t}$ represents transmitting antenna gain;

$G_{r}$ represents receiving antenna gain;

$A_{e}=G_{r} \lambda^{2} / 4 \pi$ is receiving antenna effective area;

$S_{r}=G_{t} P_{t} / 4 \pi d^{2}$ is signal power density at the receiving antenna.

If the propagating radio waves reach an surface that is larger than the radio wavelength, then the wave partially reflects and partially penetrates the obstacle material. In case the material is the perfect conductor, the wave reflects into the primary environment without the energy loss. Of course, in this case the reflection law applies. In general, the electromagnetic field strength of the reflected and passing waves can be expressed by the Fresnel reflect and transfer coefficients that depend on the material properties, the wave polarization, the angle of radio wave impact and the frequency. A more detailed analysis of the effect of reflection can be found in [11]. The reflection significantly affects the radio frequency signal propagation in the indoor environment.

The diffraction occurs when there is no direct path (lineof-sight) for the radio waves between the transmitter and the receiver antennas - the transmission path is limited by an obstacle. Due to the diffraction, in the shade of the perfect obstacle with the limited dimensions, the radio signal strength has a non-zero value. The effect of the signal diffraction can be neglected at high frequencies.

Signal scattering is present in cases where the electromagnetic waves pass through or reflect from the objects that have comparable or smaller dimensions than the wavelength. Scattered waves occur when rough surfaces and small objects appear in the space of the transmission channel. The signal level varies at the receiver antenna because of the signal scattering and the levels are usually different from the values predicted by the propagation models that even take into account the path-loss, reflection and diffraction.

Remark 2.2: Note that the larger signal energy is scattered, the more the energy of the reflected waves is reduced. All the above mentioned effects, affect the propagation of the electromagnetic waves indoors. The way how they impact depends on the signal wavelength and the environmental properties and circumstances.

\section{CASE STUdY}

To investigate the case of the electromagnetic wave propagation we used the hallway scenario illustrated at the Fig. 2. The antennas (the transmitter and the receiver antenna) have been placed at the center line of the corridor in the height $80 \mathrm{~cm}$ above the floor.

For measurements we used the system-on-chip solution based on TI CC2511 (see Fig. 1) that operates in the ISM band starting at the frequency equal to $2.400 \mathrm{GHz}$ and ending at $2.4835 \mathrm{GHz}$. This ultra-low power solution integrates a fullspeed USB controller, $\mathrm{I}^{2} \mathrm{~S}$ interface USART and 12-bit A/D converter. However, more important information for us are the radio peripheral attributes. Besides the mentioned frequency range, the radio solution is capable of 2FSK, GFSK and MSK 


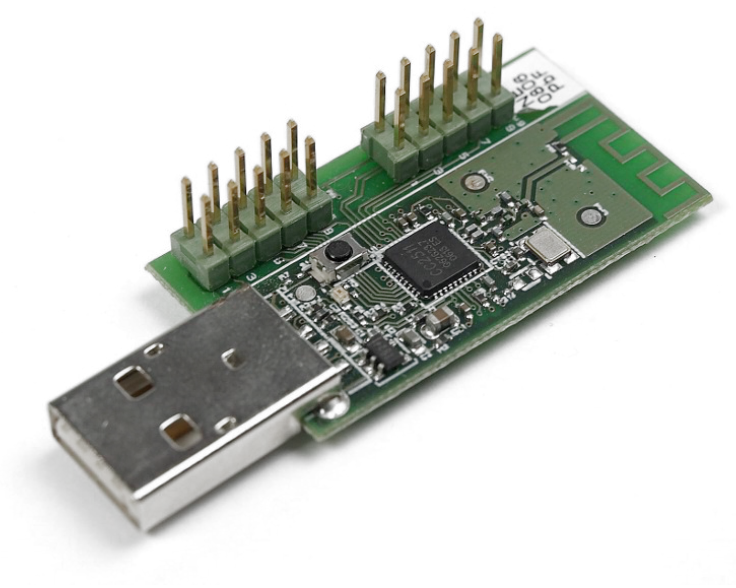

Fig. 1. Wireless system-on-chip used for experimental results

modulation techniques, programmable transmission power up to $+1 \mathrm{dBm}$, high receiver sensitivity $(-100 \mathrm{dBm}$ in average) along with the 128-bit AES security co-processor.

In the first step, we will try to describe the propagation of the electromagnetic waves with Five-Ray propagation model (see Fig. 3), in which we consider a simple reflections from the side walls, floor and ceiling of the hall.

Then the impulse response function of the transmission channel can be written in the form:

$$
\begin{aligned}
h(t) & =A_{1} \cdot \sigma\left(t-T_{1}\right)+A_{2} \cdot \delta\left(t-T_{2}\right)+ \\
& +A_{3} \cdot \delta\left(t-T_{3}\right)+A_{4} \cdot \delta\left(t-T_{4}\right)+A_{5} \cdot \delta\left(t-T_{5}\right)
\end{aligned}
$$

where an expression $A_{1} \cdot \sigma\left(t-T_{1}\right)$ represents a received signal portion (from line-of-sight);

expressions $A_{2} \cdot \delta\left(t-T_{2}\right), A_{3} \cdot \delta\left(t-T_{3}\right)$ and $A_{4} \cdot \delta\left(t-T_{4}\right)$ represent reflected signal portions from the both side walls and the hallway floor;

$A_{5} \cdot \delta\left(t-T_{5}\right)$ represents a reflected contribution from the hallway ceiling.

Given the same length of the transmission path, it is possible to assume that the reflections from the both side walls and the floor have the same signal time delay $\left(T_{2}=T_{3}=T_{4}\right)$. Then, it is possible to rewrite (2) into the following form:

$$
h(t)=A_{1} \cdot \sigma\left(t-T_{1}\right)+A_{v} \cdot \delta\left(t-T_{2}\right)+A_{5} \cdot \delta\left(t-T_{5}\right)
$$

where $A_{v}=A_{2}+A_{3}+A_{4}$.

Then, the frequency response of the transmission channel is:

$$
H(j \omega)=A_{1} e^{-j T_{1} \omega}+A_{v} e^{-j T_{2} \omega}+A_{5} e^{-j T_{5} \omega} .
$$

For the times $T_{i}$ aplies:

$$
T_{1}(d)=\frac{d}{3 \cdot 10^{8}} \quad[s]
$$

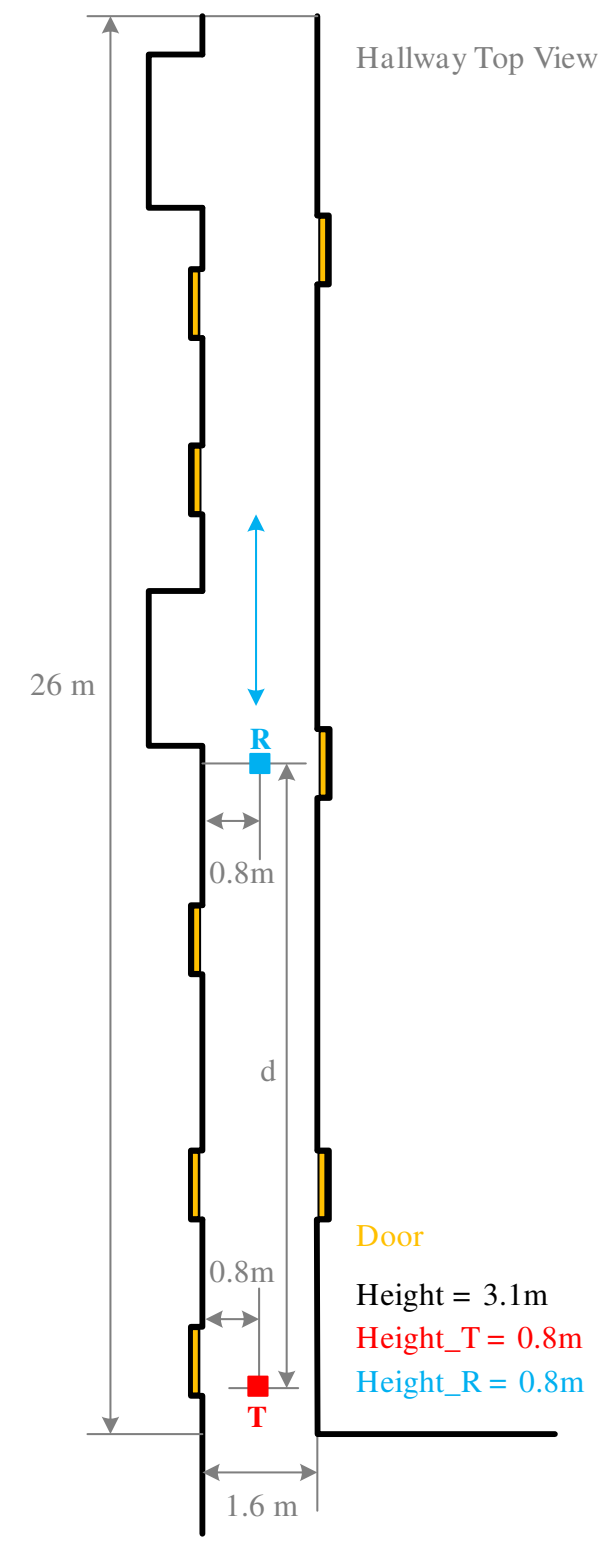

Fig. 2. Topology of the measurement indoor environment

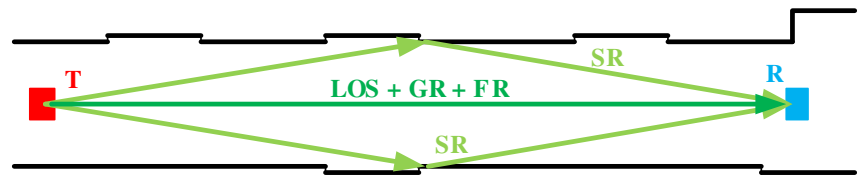

Fig. 3. Five-Ray propagation model illustration 


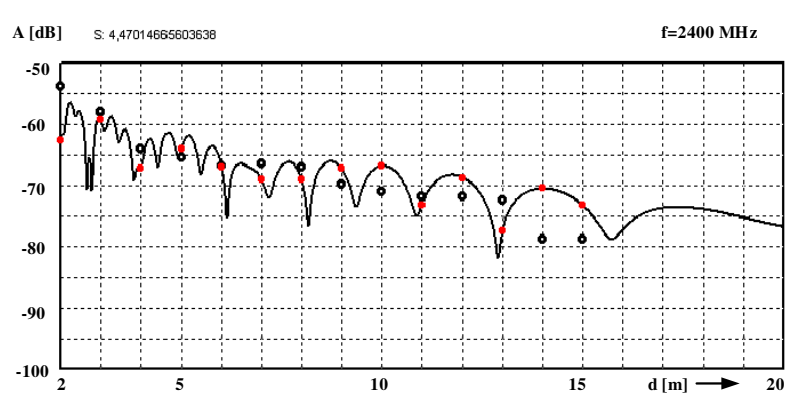

Fig. 4. Transmission power dependency on the distance $d$

$$
\begin{aligned}
& T_{2}(d)=\frac{\sqrt{d^{2}+4 \cdot 0.8^{2}}}{3 \cdot 10^{8}} \quad[s], \\
& T_{5}(d)=\frac{\sqrt{d^{2}+4 \cdot 2.3^{2}}}{3 \cdot 10^{8}} \quad[s],
\end{aligned}
$$

With respect to the (1) and if assuming that the antennas gain equal 1 , it is possible to write for $A_{1}$ the following:

$$
A_{1}=C \cdot\left(\frac{\lambda}{4 \pi d}\right)^{2},
$$

where the constant $C$ depends on the antennas gain (transmitting and receiving antenna).

For all reflected radio waves applies:

$$
A_{i}=K_{i} \cdot C \cdot\left(\frac{\lambda}{4 \pi d_{i}}\right)^{2},
$$

where the coefficient $K_{i}$ is the reflection coefficient that depends on reflection plane material, reflection plane surface, the frequency, etc.)

Based on the mathematical representation of the Five-Ray signal propagation model, the experimental results have been acquired. These results show the dependency of the channel path-loss on the distance between the receiver and the transmitter. The graphical representation of the data with reflection coefficient values set to 0.5 and the frequency $2400 \mathrm{MHz}$ is shown on the Fig. 4.At the same time, the Fig. 4 shows the measured values in the specific measurement points (black circles). The red dots represent the values predicted by the propagation model at the specific measurement points.

As the measure of the propagation model match with the experimental results, we selected the relationship (10). Having the zero-mean error of the propagation model this relationship represents a selective standard error deviation of the model.

$$
S=\sqrt{\frac{1}{N-1} \sum_{i=1}^{N}\left(M_{i}-M_{o}\right)^{2}},
$$

where $M_{i}$ represent the measured values at the specific measurement points;

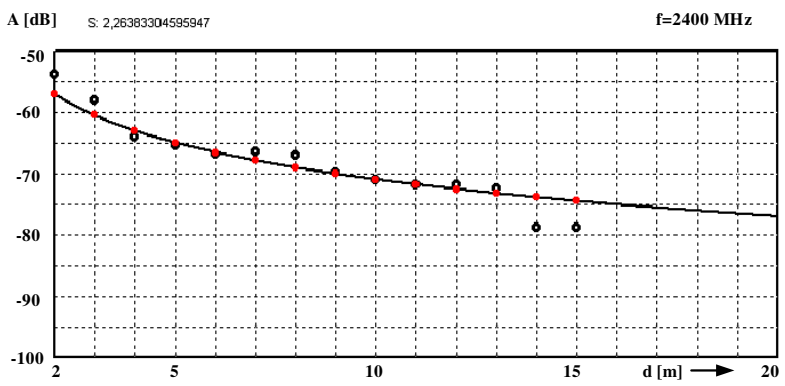

Fig. 5. Transmission power path-loss dependency on the distance $d$

$M_{o_{i}}$ represent the model calculated values at the specific measurement points.

When comparing the measured values with the values obtained from the Five-Ray propagation model, we received an average standard deviation value equal $S \approx 4.5 d B$. We can conclude that the indoor environment is too complex for the transmission path with strong scattering and multiple electromagnetic waves reflections. Therefore, it is impossible to describe these effects using any analytical propagation model, unlike the possibility in case of the urban environment (urban mikro-cell).

Let's analyze an option of describing the electromagnetic wave propagation through the Friis equation to calculate the path-loss of the electromagnetic waves in the free space. Then, the mathematical model describing the signal path-loss dependency on the distance between the transmitting and the receiving antenna will be in the form:

$$
\begin{aligned}
A_{d B}(d)=10 \log _{10} & \left(\frac{P_{r}}{P_{t}}\right)= \\
& =20 \log _{10}(\lambda)-20 \log _{10}(d)+K,
\end{aligned}
$$

where $P_{t}$ is the transmission power;

$P_{r}$ is the power at the receiving antenna output;

$K$ is a constant representing the antennas' parameters (receiving and transmitting antenna; $K=20 \log _{10}(4 \pi)-$ $\left.10 \log _{10}\left(G_{r} G_{t}\right)\right)$

A graphical illustration of the $2400 \mathrm{MHz}$ electromagnetic wave path-loss dependency on the distance between the transmitting and receiving antenna $d$ is shown in the Fig. 5. Similarly to the Fig. 4, this figure shows the measured values in each specific measurement point (black circles) and the propagation model calculated values (red dots). The propagation model is defined in (11).

Likewise, as in the previous case of the Five-Ray propagation model, for this one we determined the standard error deviation of the model $S=2.26 \mathrm{~dB}$ based on the model calculated values and measured values.

According to [11], in most cases, we can use a simplified model of the wave propagation in the form:

$$
A(d)=K \cdot\left(\frac{d_{0}}{d}\right)^{\gamma}
$$


TABLE I

TYPICAL VALUES OF $\gamma$ EXPONENT FOR DIFFERENT ENVIRONMENTS

\begin{tabular}{|l|c|}
\hline \multicolumn{1}{|c|}{ Environment } & $\gamma$ value \\
\hline Urban area (macro-cells) & $3.7-6.5$ \\
\hline Urban area (micro-cells) & $2.7-3.5$ \\
\hline Office space (same floor) & $1.6-3.5$ \\
\hline Office space (different floors) & $2-6$ \\
\hline Commerce & $1.8-2.2$ \\
\hline Industrial & $1.6-3.3$ \\
\hline Household & 3 \\
\hline
\end{tabular}

$$
A_{d B}(d)=10 \log K-10 \gamma \log \left(\frac{d}{d_{0}}\right),
$$

where $K$ is a function of the average environment path-loss and takes into account the characteristics of the antennas;

$d_{0}$ is the reference distance that for the indoor environment should be in the interval $1-10 \mathrm{~m}$. (for $2.4 \mathrm{GHz}$ frequency, it is recommended to set the value $d_{0}$ at the lower interval boundary, for outdoor environment $d_{0}$ should be from the interval $10-100 \mathrm{~m}$ ).

The value of the coefficient $K$ may be set approximately in the reference distance $d_{0}$ based on the Friis relationship:

$$
K=\left(\frac{\lambda}{4 \pi d_{0}}\right)^{2}
$$

Better way of the path-loss value $K\left(d_{0}\right)$ determination in the reference distance for certain conditions is to measure it. Similarly, also the $\gamma$ value depends on the specific environmental conditions and can be set by measurements. The easiest way is to minimize the sum of difference squares of the measurements in the specific measurement points $d_{i}$ and model predicted values $\left(\sum\left(M_{o}\left(d_{i}\right)-M_{i}\right)^{2}\right)$, where $M_{i}$ represent the measured values).

Available literature on propagation models gives approximate values of the $\gamma$ exponent - Table I.

Considering that the random environmental factors contribute to the path-loss propagation given by the value $E_{d B}$, then the relationship (9) turns into the following form:

$$
A_{d B}(d)=10 \log K-10 \gamma \log \left(\frac{d}{d_{0}}\right)+E_{d B},
$$

where $E_{d B}$ is a random variable with a Gaussian distribution with zero mean and standard deviation $S$. Then, based on the experimental measurements it is possible to create the mathematical model (9) that minimizes the sum of difference squares of the measured values and the model calculated values. It can be proved that for this criteria based on the $N+1$ different measurements in different distances $d_{i}=0,1,2, \ldots, N$ it is possible to determine $\gamma$ value as follows:

$$
\gamma=\frac{\sum_{1}^{N}\left(K\left(d_{0}\right)-M\left(d_{i}\right) \cdot \log _{10}\left(\frac{d_{i}}{d_{0}}\right)\right)}{10 \sum_{1}^{N}\left(\log _{10}\left(\frac{d_{i}}{d_{0}}\right)\right)^{2}},
$$

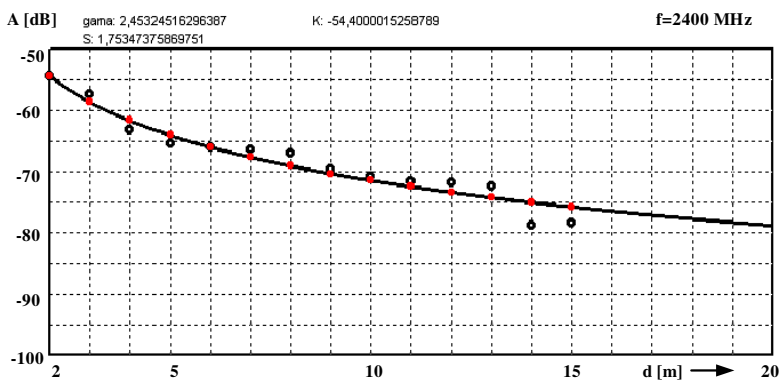

Fig. 6. Transmission power path-loss dependency on the distance $d$ (black circles represent the measured values and the red dots represent the predicted values from the propagation model (17))

where $K\left(d_{0}\right.$ is the path-loss in the reference distance $d_{0}$ (it can be obtained by measurements eventually it is possible to determine its approximate value according to (10),

$M\left(d_{i}\right)$ is the measured path-loss value at a distance $d_{i}$ from the transmitter.

Remark 3.1: Note that the above model is applicable only for distances $d>d_{0}$.

Based on the (11) and (12) a transmission channel model can be written in the form:

$$
A_{d B}(d)=C-10 \gamma \log \left(\frac{d}{2}\right),
$$

where the reference distance has been chosen $d_{0}=2 m$. Fig. 6 illustrates the obtained results.

Similarly, as in the previous cases, based on the measured and calculated values the standard error deviation of the model has been determined $S=1.753 d B$. It is obvious that the present model is the most appropriate for determining approximate path-loss of radio signal propagation in indoor environments.

Remark 3.2: However, in practical implementation of the latter model, prior to implementation, it is necessary to perform sample measurements in the the investigated environment.

During the verification of the model, there have been measurements in 9 frequency channels performed. The investigated channels were spaced by $10 \mathrm{MHz}$ from the range beginning at $2400 \mathrm{MHz}$ up to $2480 \mathrm{MHz}$. The obtained parameters of the propagation model for different frequency channels are shown in Table II.

Remark 3.3: Propagation model used in Table II is in the form: $A_{d B}(d)=C-10 \gamma \log _{10}(d / 2), P_{t}=$ $+1 d B m, 2 F S K$ modulation.

It is obvious that for each channel in the investigated ISM frequency band, we receive a propagation model characterized by different parameters. This situation arises from the fact that the values obtained during the measurements in each channel are slightly different. The path-loss in the individual channels (1-9) at distances of $2-15 \mathrm{~m}$ is illustrated in Fig. 7. 
TABLE II

PROPAGATION MODEL PARAMETERS

\begin{tabular}{|l|c|c|c|}
\hline Channel & Frequency $[\mathbf{M H z}]$ & $\gamma$ & C \\
\hline 1 & 2400 & 2.5492 & -53.9 \\
\hline 2 & 2410 & 2.4096 & -53.5 \\
\hline 3 & 2420 & 2.9357 & $-50,5$ \\
\hline 4 & 2430 & 2.6661 & -53.0 \\
\hline 5 & 2440 & 2.6640 & -50.1 \\
\hline 6 & 2450 & 2.5445 & -51.8 \\
\hline 7 & 2460 & 2.6750 & -49.3 \\
\hline 8 & 2470 & 3.0334 & -50.1 \\
\hline 9 & 2480 & 3.4085 & -49.7 \\
\hline
\end{tabular}

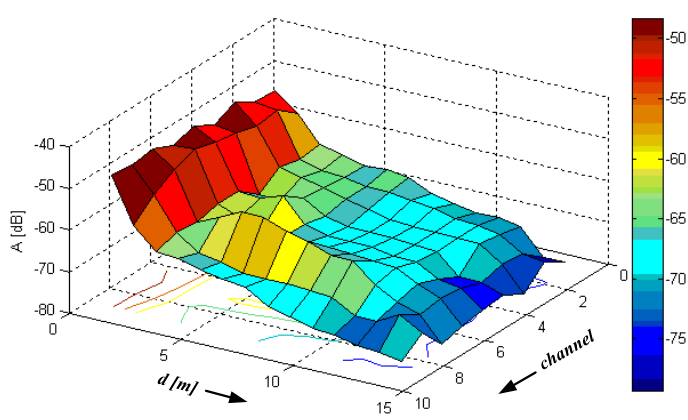

Fig. 7. Measured channel path-loss dependency on the distance $d$ varying from $2 m$ to $15 m$

The situation where each channel is characterized by a model with optimal $\gamma$ parameter and $C$ according to Table II is illustrated in Fig. 8. This figure models path-loss propagation in the $2400-2480 \mathrm{MHz}$ frequency range (channels 1-9).

Remark 3.4: Note that the standard error deviation of the model has been $S=2.46 \mathrm{~dB}$.

Let's simplify rather complicated approach for the modeling of the wave propagation that uses different models for every

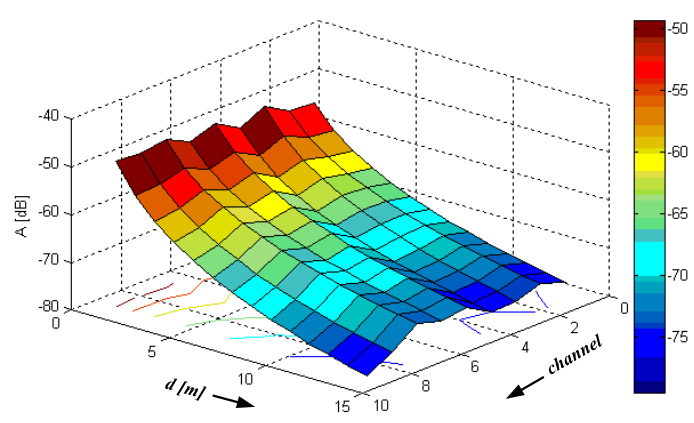

Fig. 8. Modeled channel path-loss dependency on the distance $d$ varying from $2 m$ to $15 m$

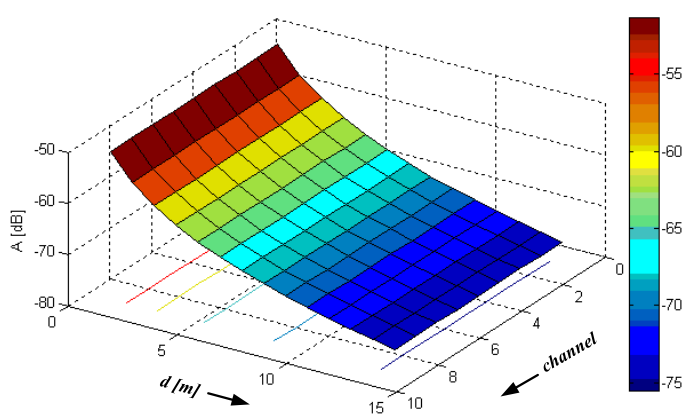

Fig. 9. Modeled path-loss dependency on the distance $d$ varying from $2 m$ to $15 \mathrm{~m}$

single channel. Let's try to substitute all 9 previous propagation models with one valid for the entire frequency range $2400-2480 \mathrm{M} \mathrm{Hz}$. During the new model description, average values of the coefficients $\gamma_{p}=2.7629$ and $C_{p}=-51.32$ are used. Then, the model characterizing the whole frequency range is in the following form (18):

$$
A_{d B}(d)=-27.629 \log \left(\frac{d}{2}\right)-51.32 .
$$

Fig. 9 is based on the model values defined by (18) and shows the dependency of path-loss propagation in the frequency range $2400-2480 \mathrm{MHz}$ at different distances $d$.

Remark 3.5: Note that the standard error deviation of the model in this last case is $S=2.9 \mathrm{~dB}$. It is obvious that there is a slight deterioration in the accuracy of the model compared to the previous one.

\section{CONCLUSION}

A comprehensive description of the electromagnetic wave propagation solve Maxwell's equations. However, this approach is computationally demanding and impractical. In real life situations, the approximate models are deployed to describe the signal propagation. These models are often based on the experimental results. This paper shows case study on indoor radio frequency signal propagation at $2.4 \mathrm{GHz}$ ISM Band supported by the related math, implementation of the propagation models and experimental results.

To investigate the case of the electromagnetic wave propagation, a specific hallway scenario has been used. The measurements were performed by the system-on-chip solution based on TI CC2511 that operates in the ISM band starting at the frequency equal to $2.400 \mathrm{GHz}$ and ending at $2.4835 \mathrm{GHz}$.

Based on the evaluation of the conducted experiments we can conclude that Multi-Ray (Five-Ray) propagation model describing a signal path-loss in indoor environment is unusable. Model based on Friis relationship is indeed easy to apply, requires no measurements, but in the case of indoor environment is applicable only in case of line-of-sight between the receiver and the transmitter. This model does not reflect the 
characteristics and topology of the environment and therefore its use is not recommended in indoor environments. Simplified model of propagation of radio waves can be used to determine the approximate communication range. The successful application requires performing the sample measurements of the path-loss.

Let's point out that the radio frequency signal propagation models are dependent on specific environmental conditions. The achieved results cannot be applied directly. It is necessary to carry out experimental measurements of path-loss at multiple locations of protected area by measuring and using the relations (15) and (16) and the optimal model parameters. Then, based on the simulation experiments with propagation models, it is possible to find a suitable deployment of the WSN nodes. Based on path-loss measurements at different distances, we can also conclude that typical signal fading due to the multi-path propagation is caused by greater distortion in the indoor environment and occur to a lesser extent than in the urban areas. However, potential signal loss cannot be suppressed by the frequency channel alternating. By changing the channels (using frequency diversity), it is possible to suppress the effect of interferences from other sources, which are now in the indoor environments strongly present at the $2.4 G H z$ ISM band.

\section{ACKNOWLEDGMENT}

This contribution/publication is the result of the project implementation Centre of excellence for systems and services of intelligent transport II. ITMS 26220120050 supported by the Research \& Development Operational Programme funded by the ERDF.
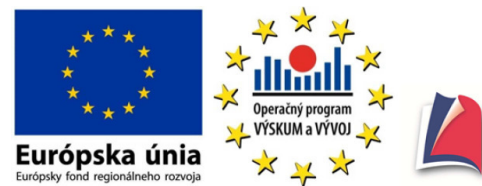

Agentúra Ministerstva školstva, vedy, viskumu a športu SR Európska únia

"Podporujeme výskumné aktivity na Slovensku/Projekt je spolufinancovaný zo zdrojov EÚ"

\section{REFERENCES}

[1] J. K. W. Wong, L. Heng, and S. W. Wang, "Intelligent building research: a review", Automation in Construction, 14.1 (2005), pp. 143-159.

[2] U.S. Department of Energy, "2011 Building Energy Data Book", March 2012.

[3] European Commission, "Report from the Commission to the European Parliament and the Council", Brussels 18.4.2013.

[4] M. Hyben, and M. Hodoň, "Low-cost Command-recognition Device", Teleinformatics Review, ISSN 2300-5149, Vol. 36, No. 3, 2013, pp. 1928.
[5] P. Ševčík, and O. Kovář, "Power unit based on supercapacitors and solar cell module", SCIECONF 2013 : the 1st international virtual scientific conference, 10.-14. June 2013: proceedings in scientific conference, ISSN 1339-3561, Žilina: University of Žilina, 2013, ISBN 978-80-5540726-5, pp. 468-471.

[6] O. Karpiš, "Software actualization in Wireless Sensor Networks", ICTIC 2012: proceedings in information and communication technologies, 1923.3.2012, Žilina: University of Žilina, 2012, ISBN 978-80-554-0513-1, pp. $51-54$.

[7] F.L. Lewis, "Wireless sensor networks", Smart environments: technologies, protocols, and applications, 2004, pp. 11-46.

[8] O. Karpiš, "System for vehicles classification and emergency vehicles detection", PDeS 2012: proceedings of 11th IFAC/IEEE international conference on programmable devices and embedded systems, Brno, May 23th - 25th, 2012, ISBN 978-3-902823-21-2, pp. 155-159.

[9] V.C.Gungor, and G.P.Hancke: Industrial Wireles Sensor Networks: Challenges, Design Principles and Technical Approaches, IEEE Transactions in Industrial Electronics, vol. 56, No.10, 2009.

[10] O. Karpiš, "Solar-cell based powering of a node for traffic monitoring", IOSR journal of engineering (IOSRJEN), ISSN 2278-8719, Vol. 3, No. 4, 2013, pp. 28-32.

[11] A. Goldsmith, "Wireless Communication", Cambridge University Press, 2005, ISBN-13 978-0-511-13675-7.

[12] M. Hudik, "Performance optimization of broadcast collective operation on multi-core cluster", ICSC 2012: tenth international conference on soft computing applied in computer and economic environments, January 20, Kunovice, Czech Republic, 2012, ISBN 978-80-7314-279-7, pp. 51-55.

[13] J. Púchyová, "Behaviour of multiagent system with defined goal", Information Sciences and Technologies: bulletin of the ACM Slovakia, ISSN 1338-1237, Vol. 5, No. 4, 2013, pp. 15-25.

[14] K. S. Low, and M. J. Er, "Wireless Sensor Networks for Industrial Environments", Computational Intelligence for Modelling, Control and Automation, 2005, 28-30 Nov. 2005, ISBN 0-7695-2504-0, pp. 271-276.

[15] M. Hodoň, and L. Čechovič, "Hall-efect based sensor for inertial navigation systems", ICTIC 2013: proceedings in conference of informatics and management sciences, 25.-29. March 2013, Žilina, Slovak Republic, ISSN 1339-231X, ISBN 978-80-554-0648-0, pp. 359-362.

[16] M. Jurečka, and L. Čechovič, "Application of pulse coupled neural network in speaker identification", MEMSTECH 2012: perspective technologies and methods in MEMS design, Lviv, Polyana, Ukraine, 18-21 April 2012, ISBN 978-617-607-229-4, pp. 125-128.

[17] J. Papán, M. Jurečka, and J. Púchyová, "WSN for forest monitoring to prevent illegal logging", FedCSIS: proceedings of the Federated conference on computer science and information systems, September 9-12, 2012, Wroclaw, Poland, ISBN 978-83-60810-51-4.

[18] J. Miček, O. Karpiš and P. Ševčík, "Body area network: analysis and application areas", International journal of engineering research and development (IJERD), ISSN 2278-800X, Vol. 6, No. 8, 2013, pp. 22-26.

[19] J. Púchyová, M. Kochláň and M. Hodoň, "Development of special smartphone-based Body Area Network: Energy Requirements", Federated conference on computer science and information systems (FedCSIS), September 8-11, 2013. Krakow, Poland, IEEE, 2013, ISBN 978 1-4673-4471-5, pp. 915-920.

[20] D. Laqua, and P. Husar, "Intelligent Power Management enables Autonomous Power Supply of Sensor Systems for Modern Prostheses", in Biomedizinische Technik/Biomedical Engineering (Impact Factor: 1.16), September, 2012, pp. 247-250. DOI:10.1515/bmt-2012-4055.

[21] A. Hofmann, D. Laqua, and P. Husar, "Piezoelectric Based Energy Management System for Powering Intelligent Implants and Prostheses", Biomedizinische Technik/Biomedical Engineering (Impact Factor: 1.16). September, 2012, pp. 263-266. DOI:10.1515/bmt-2012-4265.

[22] J. Miček and M. Kochláň, "Energy-efficient communication systems of wireless sensor networks", Studia informatica universalis, ISSN 16217545, 2013, Vol. 11, No. 1, pp. 69-86.

[23] D. Suciu, "A Study of RF Link and Coverage in ZigBee", Scientific Bulletin of the "Petru Maior", University of Targu Mures, Vol.7, No.1, 2010, ISSN 1841-9267. 